\title{
Computed tomography (CT) and magnetic resonance imaging (MRI) of diffuse liver disease: a multiparametric predictive modelling algorithm can aid categorization of liver parenchyma
}

\author{
Ricardo Donners ${ }^{1}$, Carmen Zaugg ${ }^{1}$, Julian E. Gehweiler ${ }^{1}$, Tuyana Boldanova ${ }^{2,3}$, Markus H. Heim ${ }^{2,3}$, \\ Luigi M. Terracciano ${ }^{4}$, Daniel T. Boll ${ }^{1}$ \\ ${ }^{1}$ Department of Radiology, University Hospital Basel, University of Basel, Basel, Switzerland; ${ }^{2}$ Division of Gastroenterology and Hepatology, \\ University Hospital Basel, Basel, Switzerland; ${ }^{3}$ Department of Biomedicine, University of Basel, Basel, Switzerland; ${ }^{4}$ Institute of Pathology, \\ University Hospital Basel, Basel, Switzerland
}

Contributions: (I) Conception and design: R Donners, C Zaugg, JE Gehweiler, DT Boll; (II) Administrative support: R Donners, MH Heim, LM Terracciano, DT Boll; (III) Provision of study materials or patients: R Donners, T Boldanova, MH Heim, LM Terracciano, DT Boll; (IV) Collection and assembly of data: R Donners, C Zaugg, T Boldanova; (V) Data analysis and interpretation: R Donners, C Zaugg, JE Gehweiler, LM Terracciano; (VI) Manuscript writing: All authors; (VII) Final approval of manuscript: All authors.

Correspondence to: Ricardo Donners, MD. Department of Radiology, University Hospital Basel, Petersgraben 4, CH-4031 Basel, Switzerland. Email: ricardo.donners@usb.ch.

Background: Liver steatosis is common and tracking disease evolution to steatohepatitis and cirrhosis is essential for risk stratification and resultant patient management. Consequently, diagnostic tools allowing categorization of liver parenchyma based on routine imaging are desirable. The study objective was to compare established mono-factorial, dynamic single parameter and iterative multiparametric routine computed tomography (CT) and magnetic resonance imaging (MRI) analyses to distinguish between liver steatosis, steatohepatitis, cirrhosis and normal liver parenchyma.

Methods: A total of 285 multi-phase contrast enhanced CT and 122 MRI studies with histopathological correlation of underlying parenchymal condition were retrospectively included. Parenchymal conditions were characterized based on CT Hounsfield units (HU) or MRI signal intensity (SI) measurements and calculated HU or SI ratios between non-contrast and contrast enhanced imaging time points. First, the diagnostic accuracy of mono-factorial analyses using established, static non-contrast HU and in- to opposed phase SI change cut-offs to distinguish between parenchymal conditions was established. Second, single dynamic discriminator analyses, with optimized non-contrast and enhancement HU and SI ratio cut-off values derived from the data, employing receiver operating characteristic (ROC) curve areas under the curve (AUCs) and the Youden index for maximum accuracy, were used for disease diagnosis. Third, multifactorial analyses, employing multiple non-contrast and contrast enhanced HU and SI ratio cut-offs in a nested, predictive-modelling algorithm were performed to distinguish between normal parenchyma, liver steatosis, steatohepatitis and cirrhosis. CT and MRI analyses were performed separately.

Results: No single CT or MRI parameter showed significant difference between all four parenchymal conditions (each $\mathrm{P}>0.05$ ). Mono-factorial static-CT-discriminator analyses identified liver steatosis with $75 \%$ accuracy. Mono-factorial MRI analyses identified steatosis with $89 \%$ accuracy. Single-dynamic CT parameter analyses identified normal parenchyma with $72 \%$ accuracy and cirrhosis with $75 \%$ accuracy. Single-dynamic MRI parameter analyses identified fatty parenchyma with $90 \%$ accuracy. Multifactorial CT analyzes identified normal parenchyma with $84 \%$, liver steatosis with $95 \%$, steatohepatitis with $95 \%$ and cirrhosis with $80 \%$ accuracy. Multifactorial predictive modelling of MRI parameters identified normal parenchyma with $79 \%$, liver steatosis with $89 \%$, steatohepatitis with $92 \%$ and cirrhosis with $89 \%$ accuracy.

Conclusions: Multiparametric analyses of quantitative measurements derived from routine CT and MRI, 
utilizing a predictive modelling algorithm, can help to distinguish between normal liver parenchyma, liver steatosis, steatohepatitis and cirrhosis.

Keywords: Liver; fatty liver; liver cirrhosis; diagnostic imaging; decision-support

Submitted Apr 07, 2021. Accepted for publication Sep 06, 2021.

doi: 10.21037/qims-21-384

View this article at: https://dx.doi.org/10.21037/qims-21-384

\section{Introduction}

Liver steatosis is the most common chronic liver disease worldwide (1). The importance of tracking disease evolution from liver steatosis to steatohepatitis and cirrhosis is widely recognized (2-4). Early diagnosis and differentiation of type and severity of underlying parenchymal liver disease is essential for risk stratification and resultant patient management. Lack of early diagnosis may result in potentially lethal complications such as portal hypertension and hepatocellular carcinoma (HCC) $(5,6)$.

Quantitative, mono-factorial threshold-based concepts for detection and follow-up of fatty liver disease have been incorporated into clinical routine imaging by utilizing either non-contrast (NC) computed tomography (CT) or chemical shift magnetic resonance imaging (MRI). A NC CT threshold of 40 Hounsfield units (HU) of liver parenchyma is commonly employed to identify steatosis $(7,8)$. Analogously, a signal loss of greater than $20 \%$ between in-phase (IP) and opposed phase (OP) (I/O) Dixon T1-weighted MRI sequences indicates fatty parenchyma. Diagnostic challenges remain, as contemporary routine imaging usually does not allow discrimination between liver steatosis and steatohepatitis, with novel, more quantitative methods being currently evaluated (9-11). The identification of cirrhosis in clinical routine is largely based on qualitative imaging features related to liver morphology, such as liver border smoothness, parenchymal nodularity and lobar hyper- and hypotrophy (12). These imaging features are reader dependent and lack sensitivity (13). Ultrasoundbased elastography (14) or magnetic resonance (MR) elastography (15) may detect and quantify liver fibrosis, but are not yet integrated into routine clinical workflows $(7,16)$. Ultimately, liver biopsy remains the reference standard for diagnosis of diffuse parenchymal liver disease, but is associated with limitations such as patient acceptance, costs and complications as well as sampling only a small area of the organ resulting in measurement bias $(17,18)$. Consequently, feasible, quantitative decision-support tools, aiding the radiologist to categorize liver parenchyma and allow follow-up evaluation resolved for the entire organ based on contemporary routine CT and MRI are desirable.

This study was designed to test the hypothesis that quantitative parameters extracted from NC and contrastenhanced CT and MRI, respectively, arranged in a nested decision tree yield higher diagnostic accuracy to categorize liver parenchyma and identify underlying liver disease than mono-factorial analyses. Thus, the purpose was to compare established mono-factorial, dynamic single parameter and iterative multiparametric CT and MRI analyses to distinguish between normal liver parenchyma, liver steatosis, steatohepatitis and cirrhosis.

\section{Methods}

\section{Study design and patient selection}

This retrospective, single-center study was conducted in accordance with the Declaration of Helsinki (as revised in 2013) and the legal regulations of the institutional research and ethics committee. Individual consent for this retrospective analysis was not necessary. A key word search in the in-house pathology data system revealed liver parenchyma biopsy data of 790 unique adult patients performed between 02/2002-05/2017. Biopsies were performed, for suspected diffuse liver disease or focal lesions. In the latter case, the routinely performed additional parenchymal biopsy remote from the liver lesion was included for analysis in this study. The pathology reports were screened for conclusive diagnoses of normal parenchyma, liver steatosis, steatohepatitis or liver cirrhosis within specified biopsied liver segments. Liver fibrosis, as one diagnostic feature of liver cirrhosis, was rated by the pathologists as none present, mild, moderate or severe 
and was staged using the METAVIR scoring system in hepatitis $\mathrm{C}$ patients and Ishak system in hepatitis B patients. Correlation of imaging parameters with fibrotic grading was beyond the scope of this study.

Correlation of patient data with the radiology information system confirmed if multiphase NC and contrast-enhanced CT or MRI were available and patients suitable for study inclusion. Study imaging inclusion criteria were: MRI protocols had to include T1 weighted gradient-echo Dixon and diffusion-weighted imaging (DWI), CT/MRI had to be performed within 12 months from the time of biopsy. In total, 245 patients with 410 imaging time points between 08/2012 and 07/2017 were included for analysis. Patient demographics and histopathological diagnoses were noted.

\section{Imaging}

CT imaging was performed on 64 and 128 multislice systems (Siemens AG, Healthineers, Erlangen, Germany) with $0.6 \mathrm{~mm}$ beam collimation at $120 \mathrm{kVp}$ and $150 \mathrm{mAs}$. Arterial (A) contrast (Ultravist $370^{\circledR}$, Bayer, Leverkusen, Germany) phase was determined through bolus-triggering when a threshold $\mathrm{HU}$ value of $120 \mathrm{HU}$ was reached in the aorta at the level of the celiac trunk. Portal venous (PV) and equilibrium (Equ) phase images were acquired 20 and $140 \mathrm{~s}$ after the A phase timepoint, respectively.

MRI was acquired on Siemens 1.5 and 3 Tesla (T) systems (Siemens Avanto, Siemens Skyra, Siemens Healthineers). Model abdominal imaging protocols included axial non- and post-contrast multiphase $\mathrm{T} 1$ volumetric interpolated breath-hold examination (VIBE) and DWI. T1 VIBE 2-point Dixon images were acquired with dedicated IP and OP echo timing using the following, exemplary imaging parameters: matrix $=320 \times 195$, slice thickness $=3 \mathrm{~mm}$, repetition time $(\mathrm{TR})=6.75 \mathrm{~ms}$, echo time $(\mathrm{TE})$ $=2.39 \mathrm{~ms}$ (at $1.5 \mathrm{~T}$ ), flip angle $=10^{\circ}$, number of averages $=1$. The image acquisition time was approximately $20 \mathrm{~s}$ per axial stack. Axial DW-images were acquired using the following, exemplary imaging parameters: matrix $=128 \times 100$, slice thickness $=5 \mathrm{~mm}, \mathrm{TE}=64 \mathrm{~ms}, \mathrm{TR}=4,400 \mathrm{~ms}$, receiver bandwidth $=2,490 \mathrm{~Hz} / \mathrm{px}$, partial Fourier $=7 / 8$, b-values $=0$ and $800 \mathrm{~s} / \mathrm{mm}^{2}$, three scan trace weighted diffusion encoding, SPAIR fat-suppression. Parallel imaging acceleration was applied to reduce distortion along the phase-encoding direction $(\mathrm{R}=2)$. The number of signal averages was 2 for b0 and 6 for b800 images.
The imaging protocol was supplemented by coronal and axial T2 weighted fast spin-echo sequences. Extracellular contrast agent gadoterate meglumine, (Dotarem ${ }^{\circledR}$, Guerbet, Villepinte, France), gadobenate dimeglumine (Multihance ${ }^{\circledR}$, Bracco, Milano, Italy), gadopentetic acid dimeglumine (Magnevist $^{\circledR}$, Bayer) and the liver-specific contrast agent gadoxetate disodium (Primovist ${ }^{\circledR}$, Bayer) were used.

\section{Image analyses}

On multiphase CT and MR images, three circular regions of interest (ROIs) were placed on consecutive imaging slices specifically outlining the Couinaud segment as given in the biopsy report on PV phase images and cloned onto the corresponding NC, A and Equ phase images. Averaged mean $\mathrm{HU}$ or signal intensity (SI) values were noted for each phase. Contrast enhancement ratios (CER) were calculated from ROI measurements as follows:

$$
\begin{array}{ll} 
& (\mathrm{A}-\mathrm{NC}) / \mathrm{NC} \text { referred to as } \mathrm{A} / \mathrm{NC}_{\mathrm{CER}} ; \\
& (\mathrm{PV}-\mathrm{NC}) / \mathrm{NC} \text { referred to as } \mathrm{PV} / \mathrm{NC}_{\mathrm{CER}} ; \\
& (\mathrm{Equ}-\mathrm{NC}) / \mathrm{NC} \text { referred to as } \mathrm{Equ} / \mathrm{NC}_{\mathrm{CER}} ; \\
& (\mathrm{A}-\mathrm{PV}) / \mathrm{PV} \text { referred to as } \mathrm{A} / \mathrm{PV}_{\mathrm{CER}} ; \\
& (\mathrm{A}-\mathrm{Equ}) / \mathrm{Equ} \text { referred to as } \mathrm{A} / \mathrm{Equ}_{\mathrm{CER}} ; \\
& (\mathrm{PV}-\mathrm{A}) / \mathrm{A} \text { referred to as } \mathrm{PV} / \mathrm{A}_{\mathrm{CER}} ; \\
& (\mathrm{PV}-\mathrm{Equ}) / \mathrm{Equ} \text { referred to as } \mathrm{PV} / \mathrm{Equ}_{\mathrm{CER}} .
\end{array}
$$

On MRI, ROIs were analogously drawn to additionally outline the same liver segments on MRI in IP and OP images and the relative SI change in percentage was calculated as $(\mathrm{OP} / \mathrm{IP}-1) \times 100$. ROIs were cloned onto the apparent diffusion coefficient (ADC) map and the average mean ADC was recorded. Liver-specific contrast agents were included in the analysis, as initial distribution in vascular and interstitial components is generally assumed to be similar to extracellular contrast agents (19).

\section{Statistical analyses}

Statistical analyses were performed using commercially available software (IBM SPSS Statistics Version 22, IBM Corp. Armonk, USA and SAS JMP, SAS Institute Inc., Cary, USA).

For the mono-factorial analyses in a first step, the single static discriminating $40 \mathrm{HU}$ cut-off and $20 \%$ chemical shift imaging signal drop were employed to identify liver steatosis and diagnostic performance was noted (8).

In a second step, single dynamic discriminator optimized cut-off values were derived from study data and employed to 
distinguish between parenchymal conditions. Multivariate analysis of variance with Bonferroni post hoc tests, accounting for multiple comparisons, were performed to compare calculated ratios between normal parenchyma, steatosis, steatohepatitis and cirrhosis for the single discriminator analyses. The same tests were used to compare CERs between extracellular and hepatocyte-specific contrast MRI. A P value $<0.05$ was deemed statistically significant for all analyses. In case of significance, receiver operating characteristic (ROC) curves were generated to determine the area under the curve (AUC). Employing the Youden index, optimized cut-off values discriminating between the underlying parenchymal conditions were derived.

For the multifactorial analyses, a predictive modelling algorithm was employed using the Prediction Profiler Module in JMP. "Normal Parenchyma", "Liver Steatosis", Steatohepatitis" and "Cirrhosis" were defined as outcome categories. The profiler created varying testing and confirmation datasets during the modeling process and calculated prediction models based on NC HU values and SI drop, as well as the seven CERs, resulting in two decision trees for CT and MRI with nested thresholds for the respective parameters in each model. Optimal partitioning of all decision trees was based on binary splitting. Contribution of each parameter to the model, ROC curve, as well as a confusion matrix to visualize the performance of each prediction model were generated. Based on each confusion table, sensitivities, specificities and accuracies were calculated. The minimum split size at each node was set at ten biopsy samples to avoid overfitting.

\section{Results}

\section{Study population}

A total of 172 males and 73 females with a mean age at biopsy of $65 \pm 11$ years (range, $18-91$ years) with 410 imaging time points were included in this study. There was no significant difference between the mean age of patients with normal liver parenchyma $(64 \pm 14$ years), liver steatosis ( $65 \pm 12$ years), steatohepatitis ( $68 \pm 10$ years) and cirrhosis (65 \pm 10 years, each $\mathrm{P}>0.098)$.

\section{$C T$}

A total of 285 CTs was included. Median time interval between biopsy and CT was 21 days. Histopathologically confirmed normal parenchyma was present in 70 , liver steatosis in 16, steatohepatitis in 23 and cirrhosis in 176 cases.

For the mono-factorial analyses using a single static discriminator: $\mathrm{NC} \mathrm{HU}$ was lower than $40 \mathrm{HU}$ in 38 patients including the diagnoses liver steatosis [5], steatohepatitis [6] and cirrhosis [27] resulting in 31\% sensitivity, $77 \%$ specificity and $75 \%$ accuracy for diagnosis of liver steatosis. Histopathologically verified fatty parenchyma was present in 99 cases (16 Steatosis, 12 Steatohepatitis, 71 cirrhosis). For identification of histopathological presence of fatty parenchyma regardless of one of the four main diagnoses sensitivity/specificity/accuracy were $32 \% / 90 \% / 78 \%$ using the static $40 \mathrm{HU}$ cut-off.

For the mono-factorial analyses using a single dynamic discriminator: Mean NC HU and CER values are summarized in Table 1. Mean NC HU was significantly higher in normal parenchyma (58 HU) compared to liver steatosis (42 HU), steatohepatitis (44 HU) and cirrhosis (50 HU, each $\mathrm{P}<0.001, \mathrm{AUC}=0.755)$. ROC analyses revealed highest diagnostic accuracy for identification of normal parenchyma at a cut-off value of $54.5 \mathrm{HU}$, yielding $68 \%$ sensitivity, $74 \%$ specificity and $72 \%$ accuracy. A $54.5 \mathrm{HU}$ cut-off identified histopathologically verified fatty parenchyma regardless of ultimate diagnosis (16 liver steatosis, 12 steatohepatitis, 35 cirrhosis) versus non-fatty parenchyma with $52 \%$ sensitivity, $85 \%$ specificity and $78 \%$ accuracy.

$\mathrm{PV} / \mathrm{Equ}_{\mathrm{CER}}$ was significantly lower in cirrhosis than in normal parenchyma, liver steatosis and steatohepatitis (each $\mathrm{P}<0.001$, AUC $=0.819$ ). A cut-off value of 12.7 identified cirrhosis with $72 \%$ sensitivity, $81 \%$ specificity and $75 \%$ accuracy. Neither NC HU nor CERs did show significant differences between steatohepatitis and steatosis (each $\mathrm{P}>0.165)$.

Multifactorial predictive modeling: the predictive modelling algorithm employed the parameters $\mathrm{NC} \mathrm{HU}$ and PV/Equ (each 5x), Equ/NC (3x), A/NC (2x), PV/NC (2x), $\mathrm{A} / \mathrm{Equ}(1 \times)$ and Equ/NC-CER $(1 \times)$ as nodal discriminators to distinguish between normal parenchyma, liver steatosis, steatohepatitis and liver cirrhosis. The abbreviated decision tree, showing the final branch output is shown in in Figure 1. The full output file of the SAS Prediction Profiler Module is shown in Figure S1. Corresponding ROC AUCs resulting from predictive modelling are depicted in Figure 2. 
Table 1 Average CT measurements

\begin{tabular}{|c|c|c|c|c|c|c|c|c|}
\hline \multirow{2}{*}{ Variables } & \multicolumn{8}{|c|}{ Parenchymal condition } \\
\hline & Mean & SD & Mean & SD & Mean & SD & Mean & SD \\
\hline Non contrast HU & 58.2 & 7.9 & 42.4 & 9.6 & 43.9 & 9.3 & 49.9 & 10.5 \\
\hline $\mathrm{A} / \mathrm{NC}_{\mathrm{CER}}$ & 32.82 & 30.74 & 24.18 & 11.70 & 37.28 & 20.87 & 46.09 & 33.14 \\
\hline $\mathrm{Equ} / \mathrm{NC}_{\mathrm{CER}}$ & 49.38 & 23.04 & 55.46 & 28.17 & 56.55 & 22.01 & 73.86 & 35.23 \\
\hline $\mathrm{A} / \mathrm{PV} \mathrm{V}_{\mathrm{CER}}$ & -24.92 & 18.05 & -32.12 & 11.28 & -25.63 & 15.34 & -21.64 & 14.58 \\
\hline $\mathrm{A} / \mathrm{Equ}_{\mathrm{CER}}$ & -9.89 & 13.35 & -10.60 & 14.88 & -5.71 & 27.35 & -15.49 & 14.80 \\
\hline $\mathrm{PV} / \mathrm{Equ}_{\mathrm{CER}}$ & 22.12 & 15.33 & 31.08 & 12.23 & 24.38 & 17.64 & 8.57 & 9.77 \\
\hline
\end{tabular}

CT, computed tomography; HU, Hounsfield unit; SD, standard deviation; NC, non-contrast; A, arterial; PV, portal venous; Equ, equilibrium; CER, contrast enhancement ratio.

The confusion matrix of the algorithm is shown in Table 2 . The split limitation did not allow for identification of steatohepatitis. When performing binary testing of normal parenchyma versus any parenchymal disease 15 splits were performed; the predictive modeling algorithm identified normal parenchyma with $67 \%$ sensitivity, $92 \%$ specificity and $86 \%$ accuracy (AUC $=0.931$ ). For prediction of cirrhosis versus any other parenchymal condition, 20 splits were performed; cirrhosis was identified with $90 \%$ sensitivity, $67 \%$ specificity and $81 \%$ accuracy (AUC $=0.900)$.

\section{MRI}

A total of 122 MRI, including diagnoses of 50 normal parenchyma, 13 liver steatosis, 13 steatohepatitis and 46 cirrhosis were included for analyses. Median time interval between biopsy and MRI was 27 days.

For the mono-factorial analyses using a single static discriminator: I/O-drop was greater than $20 \%$ in 19 cases (1 normal, 9 liver steatosis, 7 steatohepatitis, 2 cirrhosis) and resulting sensitivity/specificity/accuracy for diagnosis of liver steatosis were 69\%/91\%/89\%. Fatty parenchyma, regardless of ultimate diagnosis, was present in 35/122 cases (13 steatosis, 8 steatohepatitis, 14 cirrhosis) and was identified with $42 \%$ sensitivity, $94 \%$ specificity and $78 \%$ accuracy using the $20 \%$ I/O-drop cut-off.

For the mono-factorial analyses using a single dynamic discriminator: mean I/O-SI drop, ADCs and CERs are summarized in Table 3. There was significant signal loss on OP compared to IP imaging in liver steatosis and steatohepatitis when compared to normal parenchyma and cirrhosis (each $\mathrm{P}<0.001)$. I/O-SI drop did not differ significantly between normal parenchyma and cirrhosis or steatosis and steatohepatitis (each $\mathrm{P}=1.0$ ). A I/O-SI drop cut-off of $14 \%$ could distinguish fatty parenchyma (liver steatosis, steatohepatitis) from cirrhosis and/or normal parenchyma with $86 \%$ sensitivity, $91 \%$ specificity and $90 \%$ accuracy. Neither CERs nor ADCs did show significant differences between the examined parenchymal categories (each $\mathrm{P} \geq 0.375$ ).

In normal parenchyma $\mathrm{A} / \mathrm{PV}_{\mathrm{CER}}$ was significantly larger when employing liver-specific (-3.8) versus extracellular contrast $(-30.5, \mathrm{P}=0.044)$ and $\mathrm{PV} / \mathrm{Equ}_{\mathrm{CER}}$ was significantly smaller in liver-specific (-9.9) versus extracellular contrast (6.9, $\mathrm{P}=0.04)$. In liver steatosis and steatohepatitis $\mathrm{A} /$ $\mathrm{PV}_{\text {CERs }}$ were significantly larger when using liver-specific contrast $(-21.7$ versus $-35.7, \mathrm{P}=0.03$ and -28.8 versus -36.9 , $\mathrm{P}=0.035$, respectively), while in cirrhosis $\mathrm{A} / \mathrm{PV}_{\mathrm{CER}}$ was significantly smaller with hepatocyte-specific contrast (-30.1 versus $21.4, \mathrm{P}=0.047)$.

Multifactorial predictive modeling: the predictive modelling algorithm performed eight splits to distinguish between the given four parenchymal conditions. I/O-SI drop (3x), ADC (3x), PV/Equ (1x), A/NC (1x) were employed as nodal discriminators to distinguish between normal parenchyma, steatosis, steatohepatitis and liver cirrhosis. The resulting nine- 


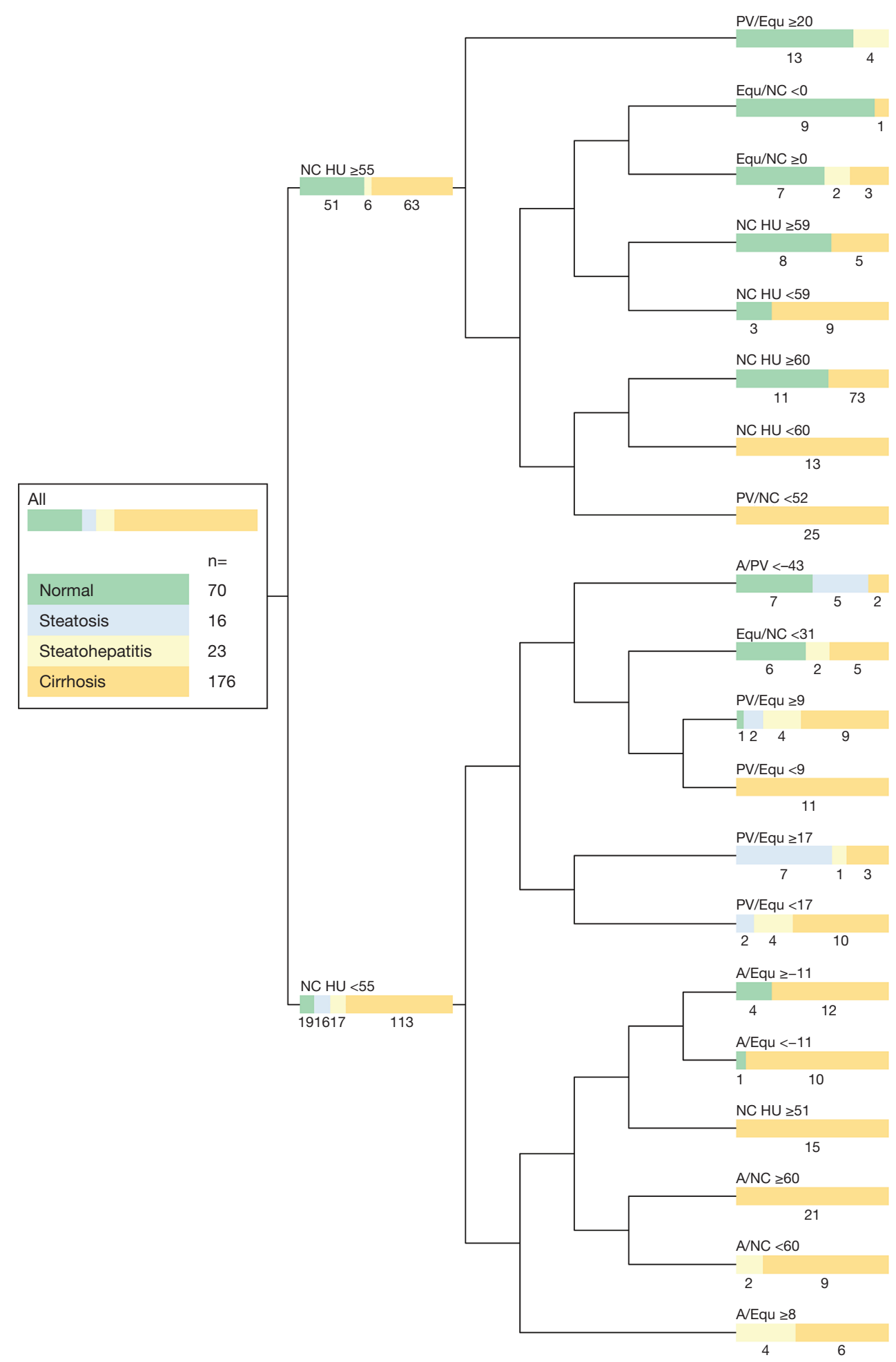

Figure 1 CT decision tree generated by the predictive modelling algorithm to distinguish between normal liver parenchyma (green), liver steatosis (blue), steatohepatitis (yellow) and cirrhosis (orange). For improved visualization, only the first and final discriminators with the resulting output count of each entity are shown. CT, computed tomography; NC, non-contrast HU; HU, Hounsfield unit; A, arterial; PV, portal venous; Equ, equilibrium contrast enhancement phase. 


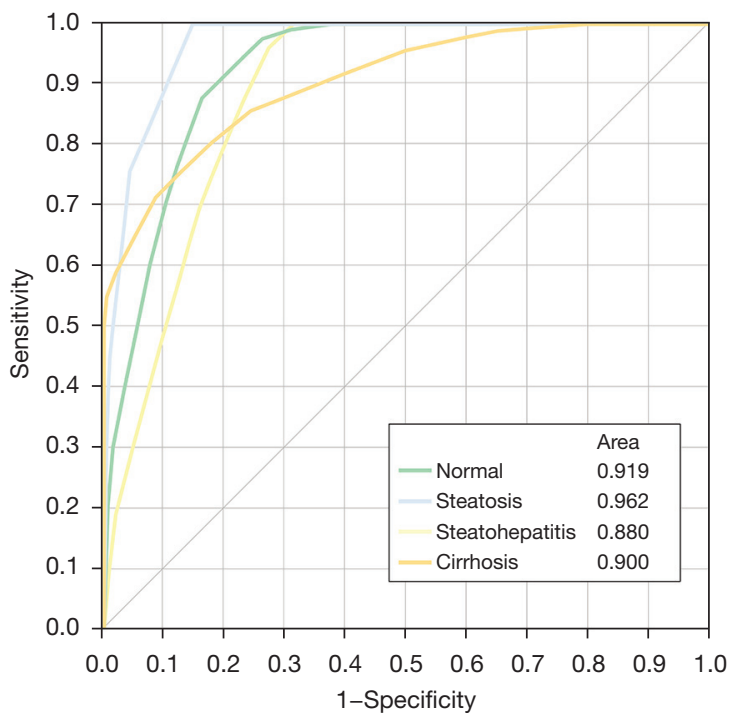

Figure 2 Receiver operator characteristics curves showing diagnostic performance of the predictive modelling algorithm employing CT non-contrast $\mathrm{HU}$ and contrast-enhancement ratios to discriminate between normal parenchyma, liver steatosis, steatohepatitis and cirrhosis simultaneously, the area under the curve is given for each parenchymal condition. CT, computed tomography; HU, Hounsfield unit.

Table 2 CT predictive modelling algorithm confusion plot

\begin{tabular}{lcccccc}
\hline \multirow{2}{*}{$\begin{array}{l}\text { Actual parenchymal } \\
\text { disease }\end{array}$} & \multicolumn{7}{c}{ Predicted count } \\
\cline { 2 - 7 } & Normal & Steatosis & Steatohepatitis & Cirrhosis & Sens. & Spec. \\
\hline Normal & 54 & 0 & 0 & 16 & $77 \%$ & $87 \%$ \\
Steatosis & 5 & 7 & 0 & 4 & $44 \%$ & $97 \%$ \\
Steatohepatitis & 6 & 1 & 0 & 16 & 0 & $100 \%$ \\
Cirrhosis & 17 & 7 & 0 & 156 & $89 \%$ & $67 \%$ \\
\hline
\end{tabular}

CT, computed tomography; Sens., sensitivity; Spec., specificity; Acc., accuracy.

Table 3 Average MRI measurements

\begin{tabular}{|c|c|c|c|c|c|c|c|c|}
\hline \multirow{3}{*}{ Variables } & \multicolumn{8}{|c|}{ Parenchymal condition } \\
\hline & \multicolumn{2}{|c|}{ Normal } & \multicolumn{2}{|c|}{ Steatosis } & \multicolumn{2}{|c|}{ Steatohepatitis } & \multicolumn{2}{|c|}{ Cirrhosis } \\
\hline & Mean & SD & Mean & SD & Mean & SD & Mean & SD \\
\hline $\mathrm{A} / \mathrm{N}_{\mathrm{CCE}} \mathrm{R}$ & 21.96 & 25.56 & 19.50 & 13.99 & 21.64 & 15.60 & 20.38 & 41.95 \\
\hline $\mathrm{PV} / \mathrm{NC}_{\mathrm{CER}}$ & 66.81 & 39.29 & 77.58 & 41.81 & 85.31 & 40.72 & 65.87 & 35.61 \\
\hline $\mathrm{A} / \mathrm{Equ}_{\mathrm{CER}}$ & -28.16 & 17.29 & -25.42 & 16.85 & -31.24 & 5.46 & -30.51 & 20.26 \\
\hline $\mathrm{PV} / \mathrm{Equ}_{\mathrm{CER}}$ & -3.26 & 20.48 & 7.59 & 17.63 & 3.67 & 11.78 & -1.86 & 10.95 \\
\hline I/O-signal drop & -1.40 & 7.94 & -35.76 & 19.28 & -31.48 & 20.36 & -3.67 & 9.46 \\
\hline ADC & $1,172.26$ & 386.58 & $1,126.52$ & 245.01 & 870.91 & 250.96 & $1,035.31$ & 355.02 \\
\hline
\end{tabular}

SD, standard deviation; NC, non-contrast; A, arterial; PV, portal venous; Equ, equilibrium; CER, contrast enhancement ratio; I/O, in-phase/ opposed phase; ADC, apparent diffusion coefficient; MRI, magnetic resonance imaging. 


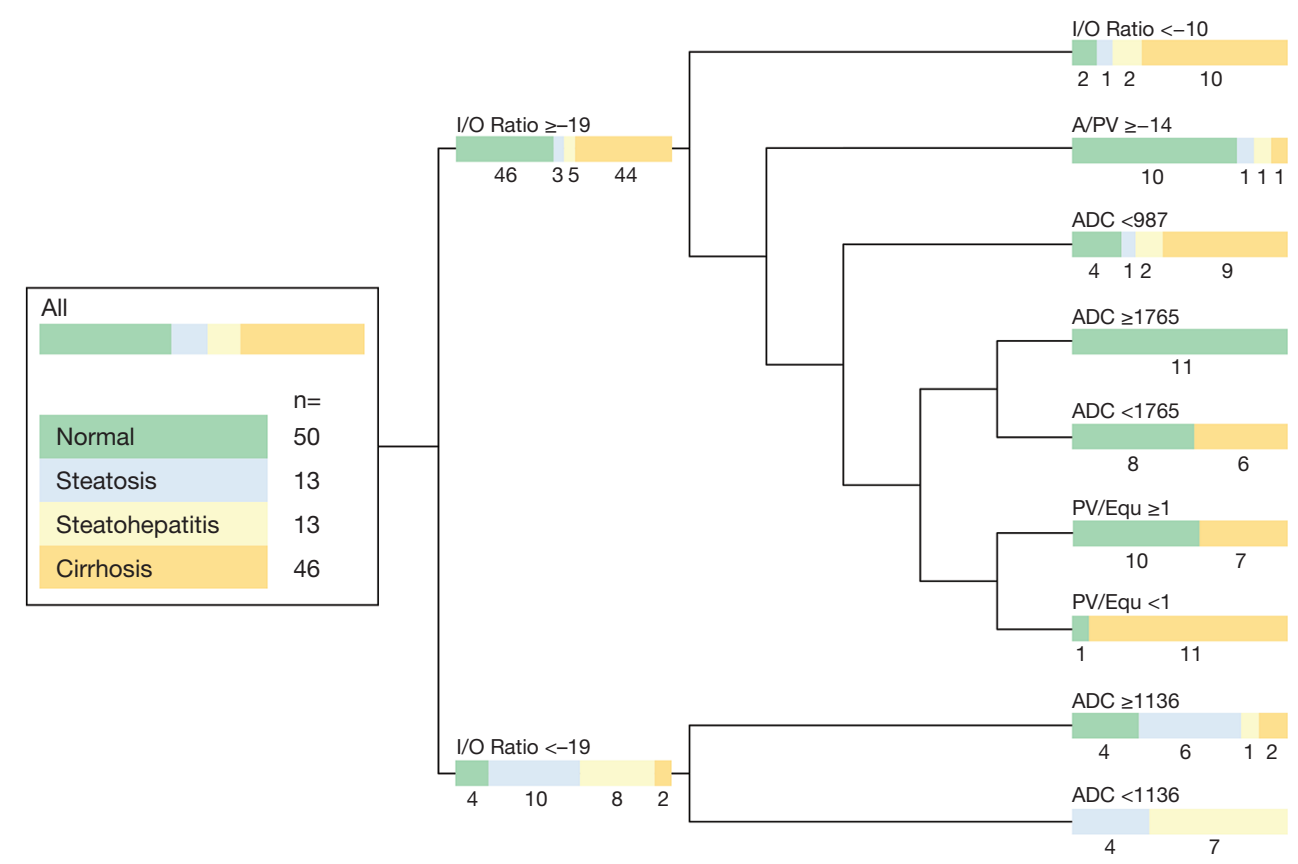

Figure 3 MRI decision tree generated by the predictive modelling algorithm to distinguish between normal liver parenchyma (green), liver steatosis (blue), steatohepatitis (yellow) and cirrhosis (orange). For improved visualization only the first and final discriminators with the resulting output count of each entity are shown. In- to opposed phase (I/O) phase ratio is given as percentage, apparent diffusion coefficient $(\mathrm{ADC})$ in $\times 10^{-3} \mathrm{~mm}^{2} / \mathrm{s}$. A, arterial; PV, portal venous; Equ, equilibrium contrast enhancement phase; MRI, magnetic resonance imaging.

tailed decision tree final branch output is shown in Figure 3. The full predictive modelling output file is shown in Figure S2. ROC AUCs derived from predictive modelling are visualized in Figure 4. The correlated confusion matrix is shown in Table 4. When performing binary testing for identification of normal parenchyma versus any parenchymal disease, the predictive modeling algorithm yielded $74 \%$ sensitivity, $83 \%$ specificity and $80 \%$ accuracy after 9 splits (AUC $=0.874$ ). For dedicated identification of cirrhosis, the algorithm took 9 iterative steps resulting in $65 \%$ sensitivity, $86 \%$ specificity and $78 \%$ accuracy (AUC $=0.861)$.

\section{Discussion}

In this pilot study, a multiparametric predictive modelling algorithm utilizing either CT NC HU values and CERs or MRI I/O-SI drop, ADC and CERs derived from routine imaging protocols allowed accurate identification of normal liver parenchyma (CT 85\%, MRI 79\% accuracy), liver steatosis (CT 95\%, MRI 89\% accuracy) or cirrhosis (CT $80 \%$, MRI $76 \%$ accuracy). In contrast, single quantitative parameter testing did not allow for simultaneous discrimination between liver steatosis, cirrhosis and normal liver parenchyma. Selective discrimination between liver steatosis and steatohepatitis and identification of steatohepatitis remain challenging, but can be partially achieved by the iterative algorithm using MRI parameters ( $92 \%$ accuracy).

This study covers a long inclusion interval from 2004 to 2017. Consequently, hardware, software and imaging protocols were subject to change and evolution, creating an inhomogeneous study group. This desired scenario resembles the diversity of scanners and software solutions utilized in radiology practices today. To be readily used in clinical practice, simple and reproducible discriminating parameters had to be identified without the need to perform major imaging protocol adjustments or integration of further imaging sequences. In contrast to previous studies, which were mostly addressing a bivariate discrimination between fatty and non-fatty or cirrhotic or non-cirrhotic parenchyma, this study was aiming to perform concurrent discrimination between the four parenchymal categories "normal", "liver steatosis", "steatohepatitis" and "liver cirrhosis" (20-22). This holistic approach is more reflective of daily practice and relevant for future machine-learning algorithms. 
The hepatic contrast-enhanced multi-phase HU values of our study group, found in normal, fatty and cirrhotic liver, are in agreement with previous literature (20-22); the same applies to the optimized NC HU cut-off levels. It needs to be noted, that the cut-off performance for identification of steatosis was relatively low in this study, when compared to previous literature: $68 \% / 74 \%$ sensitivity/specificity at $54.5 \mathrm{HU}$ in our study versus $100 \% / 95 \%$ sensitivity/

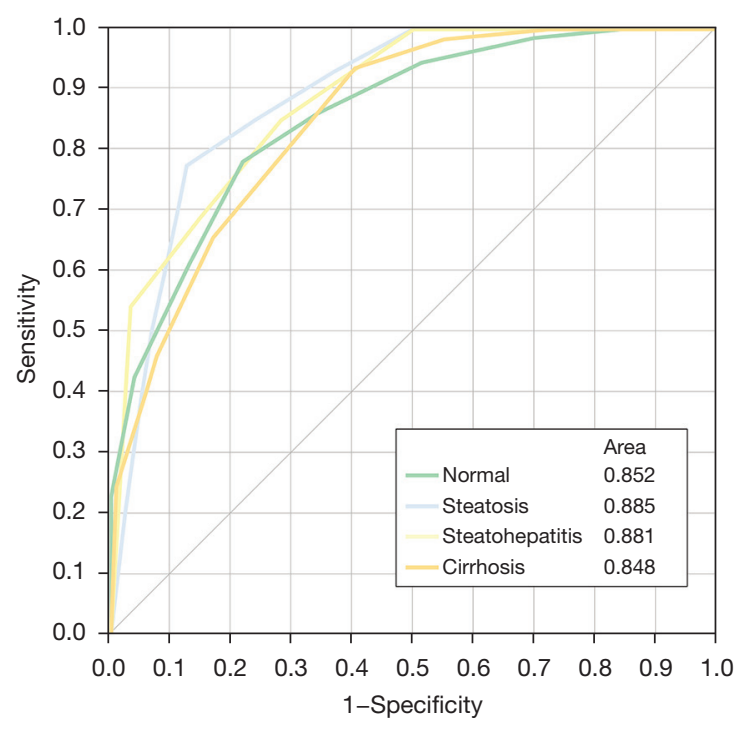

Figure 4 Receiver operator characteristics curves showing diagnostic performance of the predictive modelling algorithm employing MRI contrast-enhancement ratios, in- opposed phase signal intensity changes and apparent diffusion coefficients to discriminate between normal parenchyma, liver steatosis, steatohepatitis and cirrhosis simultaneously, the area under the curve is given for each parenchymal condition. MRI, magnetic resonance imaging. specificity at a $58 \mathrm{HU}$ cut-off (22). This may be attributed to greater heterogeneity of our study group and comparison of a bivariate versus multivariate discrimination. Application of solitary CERs to identify and distinguish between diffuse liver diseases have not been previously described. We identified $\mathrm{PV} / \mathrm{Equ}_{\mathrm{CNR}}$ to be a useful CT parameter, showing significantly smaller values in cirrhosis. This is likely due to the microscopic changes in cirrhosis: fibrosis causes an increase of interstitial space leading to an increased transfer distance between vessel and liver parenchyma (23). Consequently, contrast dynamics are delayed resulting in decreased PV and increased Equ phase attenuation (24). Cirrhosis, defined by changes of parenchymal organization, is hypothesized to lead to significantly altered liver parenchyma DWI characteristics, but the significant differences in ADC values between normal and cirrhotic parenchyma identified in previous studies could not be replicated in our population $(25,26)$.

The benefits of a quantitative approach for tissue characterization are well established (27). Especially in the context of diffuse parenchymal liver disease, which may spread unevenly (18), a definite imaging diagnosis is desirable. A liver biopsy samples only a fraction of the organ and although it is a generally safe procedure adverse events may occur, bleeding being the most important one. The iterative prediction algorithm utilized in this study can give decision-support for the radiologist regarding liver parenchyma evaluation, but did not achieve perfect accuracy distinguishing between the four included conditions. We acknowledge that this study cannot provide definite cut-off values to be used for parenchymal stratification. The algorithm's performance was limited by the minimum split size at each node of the decision tree, preventing further and improved disease discrimination.

Table 4 MRI predictive modelling algorithm confusion plot

\begin{tabular}{|c|c|c|c|c|c|c|c|}
\hline $\begin{array}{l}\text { Actual parenchymal } \\
\text { disease }\end{array}$ & \multicolumn{7}{|c|}{ Predicted count } \\
\hline Normal & 37 & 4 & 0 & 9 & $74 \%$ & $82 \%$ & $79 \%$ \\
\hline Steatosis & 1 & 6 & 4 & 2 & $50 \%$ & $87 \%$ & $89 \%$ \\
\hline Steatohepatitis & 1 & 1 & 7 & 4 & $54 \%$ & $96 \%$ & $92 \%$ \\
\hline
\end{tabular}

MRI, magnetic resonance imaging; Sens., sensitivity; Spec., specificity; Acc., accuracy. 
This may explain the apparent superior diagnostic performance of CT versus MRI parameters, as more CTs were available in this study. Smaller minimum split sizes will result in overfitting. Nevertheless, it was shown that the algorithm outperformed categorization based on optimized single parameters and clinically acknowledged cut-offs. The benefit of the utilized algorithm is that when applied, it improves with growing sample sizes and may be used in unison with an automated liver segmentation (28). This can allow for automatic highlighting of areas of potentially pathologic parenchyma and suggestion of underlying disease. In the future, threshold based predictive algorithms, automatically employed in realtime and superimposed on segmentation of routine CT and MRI can aid liver parenchyma categorization and suggest voxels and organ areas suspicious for disease to the reporting radiologist, and this study serves as a first step to build upon. There are several study limitations. First, it is retrospective study with a long inclusion interval and resultant selection bias. Second, different hardware and contrast agents were used. We included extracellular and liver-specific MRI contrast agents as they are generally assumed to follow a similar, initial distribution pattern. Although we found significant difference for a minority of CERs depending on the contrast agent, we included liver-specific contrast MRIs for analysis, as differences are likely attributed to the general study cohort heterogeneity. Nevertheless, a dedicated reproducibility study comparing early enhancement patterns using liver-specific and extracellular contrast agents in a controlled setting may be useful to test the general assumption of similar distribution. Third, liver biopsies are the reference standard for diagnosis of parenchymal liver disease, but sample only a small tissue volume, which may not always resemble the gross organ. Resected organs were not available for analysis. Finally, biopsies are mostly performed to diagnose liver cirrhosis and related pathologies. Consequently, cirrhosis accounts for more than $50 \%$ of pathology specimens in this this study and, by comparison, liver steatosis and steatohepatitis are represented in small numbers. This creates spectrum bias.

In summary, multiparametric analyses of quantitative measurements derived from routine CT and MRI utilizing a predictive modelling algorithm can help to distinguish between normal liver parenchyma, liver steatosis, steatohepatitis and cirrhosis. In this pilot study, this approach provides better accuracy than single discriminator analyses with fixed or dynamic cut-off values.

\section{Acknowledgments}

Funding: This research was partly supported by the "Foundation of the Swiss Society of Radiology for Research, Postgraduate and Continuing Medical Education" and "Research Fund for excellent Junior Researchers of the University of Basel". Sponsoring bodies had no impact on study design; in the collection, analysis and interpretation of data; in the writing of the report; or in the decision to submit the article for publication. The views expressed in this manuscript are exclusively those of the authors.

\section{Footnote}

Conflicts of Interest: All authors have completed the ICMJE uniform disclosure form (available at https://dx.doi. org/10.21037/qims-21-384). The authors have no conflicts of interest to declare.

Ethical Statement: The authors are accountable for all aspects of the work in ensuring that questions related to the accuracy or integrity of any part of the work are appropriately investigated and resolved. The study conformed to the provisions of the Declaration of Helsinki (as revised in 2013). This retrospective, single-center study was conducted according to the legal regulations of the institutional research and ethics committee of the University of Basel. Individual consent for this retrospective analysis was not necessary.

Open Access Statement: This is an Open Access article distributed in accordance with the Creative Commons Attribution-NonCommercial-NoDerivs 4.0 International License (CC BY-NC-ND 4.0), which permits the noncommercial replication and distribution of the article with the strict proviso that no changes or edits are made and the original work is properly cited (including links to both the formal publication through the relevant DOI and the license). See: https://creativecommons.org/licenses/by-nc$\mathrm{nd} / 4.0 /$.

\section{References}

1. Adams LA, Lindor KD. Nonalcoholic fatty liver disease. Ann Epidemiol 2007;17:863-9.

2. Wong VW, Wong GL, Choi PC, Chan AW, Li MK, Chan HY, Chim AM, Yu J, Sung JJ, Chan HL. Disease progression of non-alcoholic fatty liver disease: a 
prospective study with paired liver biopsies at 3 years. Gut 2010;59:969-74.

3. Adams LA, Sanderson S, Lindor KD, Angulo P. The histological course of nonalcoholic fatty liver disease: a longitudinal study of 103 patients with sequential liver biopsies. J Hepatol 2005;42:132-8.

4. Hussain SM, Reinhold C, Mitchell DG. Cirrhosis and lesion characterization at MR imaging. Radiographics 2009;29:1637-52.

5. Garcia-Tsao G. Current management of the complications of cirrhosis and portal hypertension: variceal hemorrhage, ascites, and spontaneous bacterial peritonitis. Gastroenterology 2001;120:726-48.

6. Nascimbeni F, Pais R, Bellentani S, Day CP, Ratziu V, Loria P, Lonardo A. From NAFLD in clinical practice to answers from guidelines. J Hepatol 2013;59:859-71.

7. Boll DT, Merkle EM. Diffuse liver disease: strategies for hepatic CT and MR imaging. Radiographics 2009;29:1591-614.

8. Kodama Y, Ng CS, Wu TT, Ayers GD, Curley SA, Abdalla EK, Vauthey JN, Charnsangavej C. Comparison of CT methods for determining the fat content of the liver. AJR Am J Roentgenol 2007;188:1307-12.

9. Bastati N, Feier D, Wibmer A, Traussnigg S, Balassy C, Tamandl D, Einspieler H, Wrba F, Trauner M, Herold C, Ba-Ssalamah A. Noninvasive differentiation of simple steatosis and steatohepatitis by using gadoxetic acidenhanced MR imaging in patients with nonalcoholic fatty liver disease: a proof-of-concept study. Radiology 2014;271:739-47.

10. Smits LP, Coolen BF, Panno MD, Runge JH, Nijhof WH, Verheij J, Nieuwdorp M, Stoker J, Beuers UH, Nederveen AJ, Stroes ES. Noninvasive Differentiation between Hepatic Steatosis and Steatohepatitis with MR Imaging Enhanced with USPIOs in Patients with Nonalcoholic Fatty Liver Disease: A Proof-of-Concept Study. Radiology 2016;278:782-91.

11. Lefebvre T, Hébert M, Bilodeau L, Sebastiani G, Cerny M, Olivié D, Gao ZH, Sylvestre MP, Cloutier G, Nguyen BN, Gilbert G, Tang A. Intravoxel incoherent motion diffusionweighted MRI for the characterization of inflammation in chronic liver disease. Eur Radiol 2021;31:1347-58.

12. Rofsky NM, Fleishaker H. CT and MRI of diffuse liver disease. Semin Ultrasound CT MR 1995;16:16-33.

13. Huber A, Ebner L, Montani M, Semmo N, Roy Choudhury K, Heverhagen J, Christe A. Computed tomography findings in liver fibrosis and cirrhosis. Swiss Med Wkly 2014;144:w13923.
14. Sandrin L, Fourquet B, Hasquenoph JM, Yon S, Fournier C, Mal F, Christidis C, Ziol M, Poulet B, Kazemi F, Beaugrand M, Palau R. Transient elastography: a new noninvasive method for assessment of hepatic fibrosis. Ultrasound Med Biol 2003;29:1705-13.

15. Rustogi R, Horowitz J, Harmath C, Wang Y, Chalian H, Ganger DR, Chen ZE, Bolster BD Jr, Shah S, Miller FH. Accuracy of MR elastography and anatomic MR imaging features in the diagnosis of severe hepatic fibrosis and cirrhosis. J Magn Reson Imaging 2012;35:1356-64.

16. Faria SC, Ganesan K, Mwangi I, Shiehmorteza M, Viamonte B, Mazhar S, Peterson M, Kono Y, Santillan C, Casola G, Sirlin CB. MR imaging of liver fibrosis: current state of the art. Radiographics 2009;29:1615-35.

17. Bravo AA, Sheth SG, Chopra S. Liver biopsy. N Engl J Med 2001;344:495-500.

18. Rockey DC, Caldwell SH, Goodman ZD, Nelson RC, Smith AD; American Association for the Study of Liver Diseases. Liver biopsy. Hepatology 2009;49:1017-44.

19. Thng CH, Koh TS, Collins D, Koh DM. Perfusion imaging in liver MRI. Magn Reson Imaging Clin N Am 2014;22:417-32.

20. Yajima Y, Narui T, Ishii M, Abe R, Ohtsuki M, Goto Y, Endo S, Yamada K, Ito M. Computed tomography in the diagnosis of fatty liver: total lipid content and computed tomography number. Tohoku J Exp Med 1982;136:337-42.

21. Limanond P, Raman SS, Lassman C, Sayre J, Ghobrial RM, Busuttil RW, Saab S, Lu DS. Macrovesicular hepatic steatosis in living related liver donors: correlation between CT and histologic findings. Radiology 2004;230:276-80.

22. Park SH, Kim PN, Kim KW, Lee SW, Yoon SE, Park SW, Ha HK, Lee MG, Hwang S, Lee SG, Yu ES, Cho EY. Macrovesicular hepatic steatosis in living liver donors: use of CT for quantitative and qualitative assessment. Radiology 2006;239:105-12.

23. Martínez-Esparza M, Tristán-Manzano M, Ruiz-Alcaraz AJ, García-Peñarrubia P. Inflammatory status in human hepatic cirrhosis. World J Gastroenterol 2015;21:11522-41.

24. Vignaux O, Legmann P, Coste J, Hoeffel C, Bonnin A. Cirrhotic liver enhancement on dual-phase helical CT: comparison with noncirrhotic livers in 146 patients. AJR Am J Roentgenol 1999;173:1193-7.

25. Taouli B, Koh DM. Diffusion-weighted MR imaging of the liver. Radiology 2010;254:47-66.

26. Fu F, Li X, Liu Q, Chen C, Bai Y, Shi D, Sang J, Wang $\mathrm{K}$, Wang M. Noninvasive DW-MRI metrics for staging hepatic fibrosis and grading inflammatory activity in patients with chronic hepatitis B. Abdom Radiol (NY) 
2021;46:1864-75.

27. Donners R, Blackledge M, Tunariu N, Messiou C, Merkle EM, Koh DM. Quantitative Whole-Body DiffusionWeighted MR Imaging. Magn Reson Imaging Clin N Am 2018;26:479-94.

Cite this article as: Donners R, Zaugg C, Gehweiler JE, Boldanova T, Heim MH, Terracciano LM, Boll DT. Computed tomography (CT) and magnetic resonance imaging (MRI) of diffuse liver disease: a multiparametric predictive modelling algorithm can aid categorization of liver parenchyma. Quant Imaging Med Surg 2022;12(2):1186-1197. doi: 10.21037/qims21-384
28. Graffy PM, Sandfort V, Summers RM, Pickhardt PJ. Automated Liver Fat Quantification at Nonenhanced Abdominal CT for Population-based Steatosis Assessment. Radiology 2019;293:334-42. 


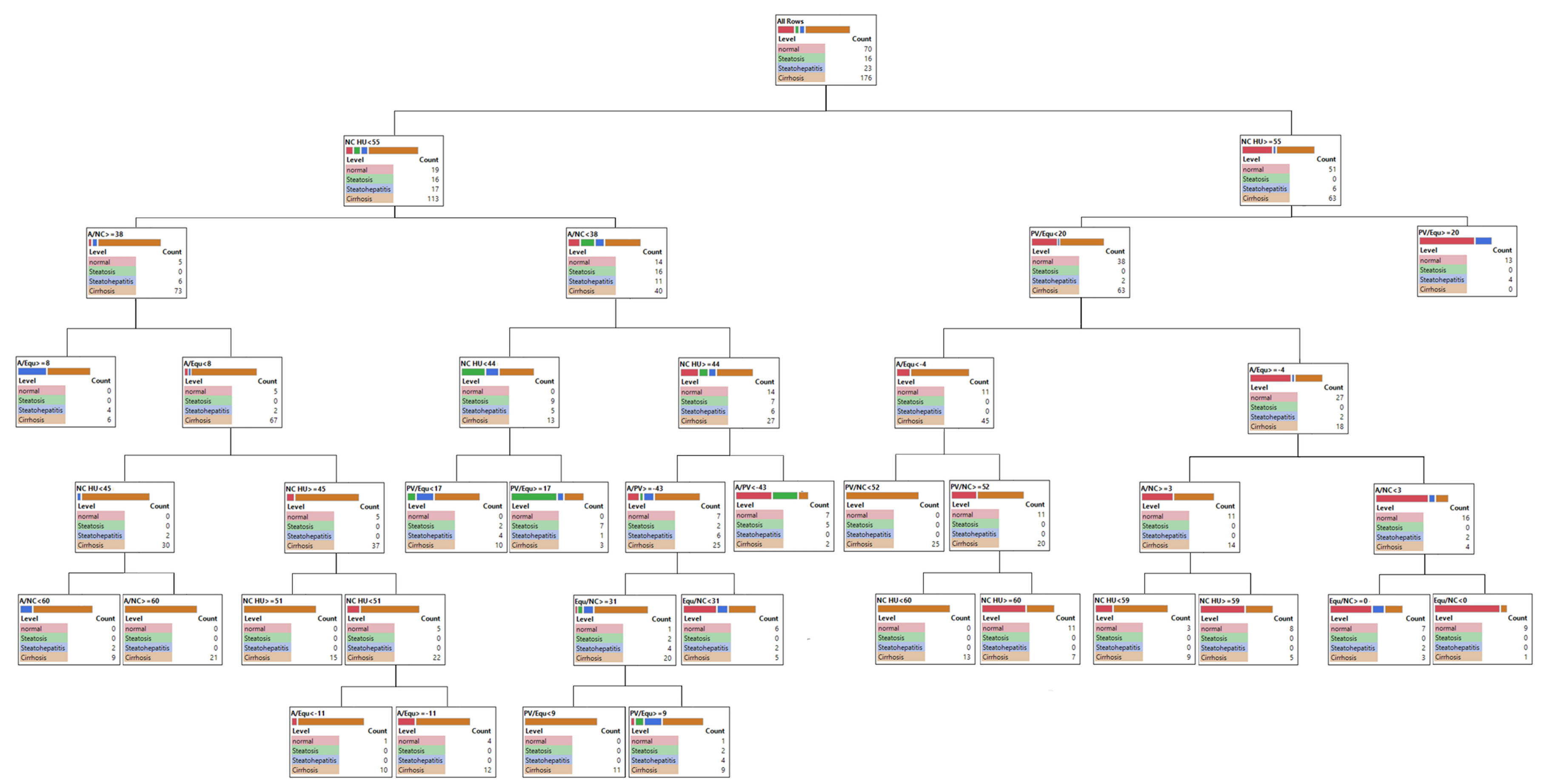

Figure S1 Full output file, decision-tree as generated by the predictive modelling algorithm for CT parameters. CT, computed tomography; NC, non-contrast HU; HU, Hounsfield unit; A, arterial; PV, portal venous; Equ, equilibrium contrast enhancement phase. 


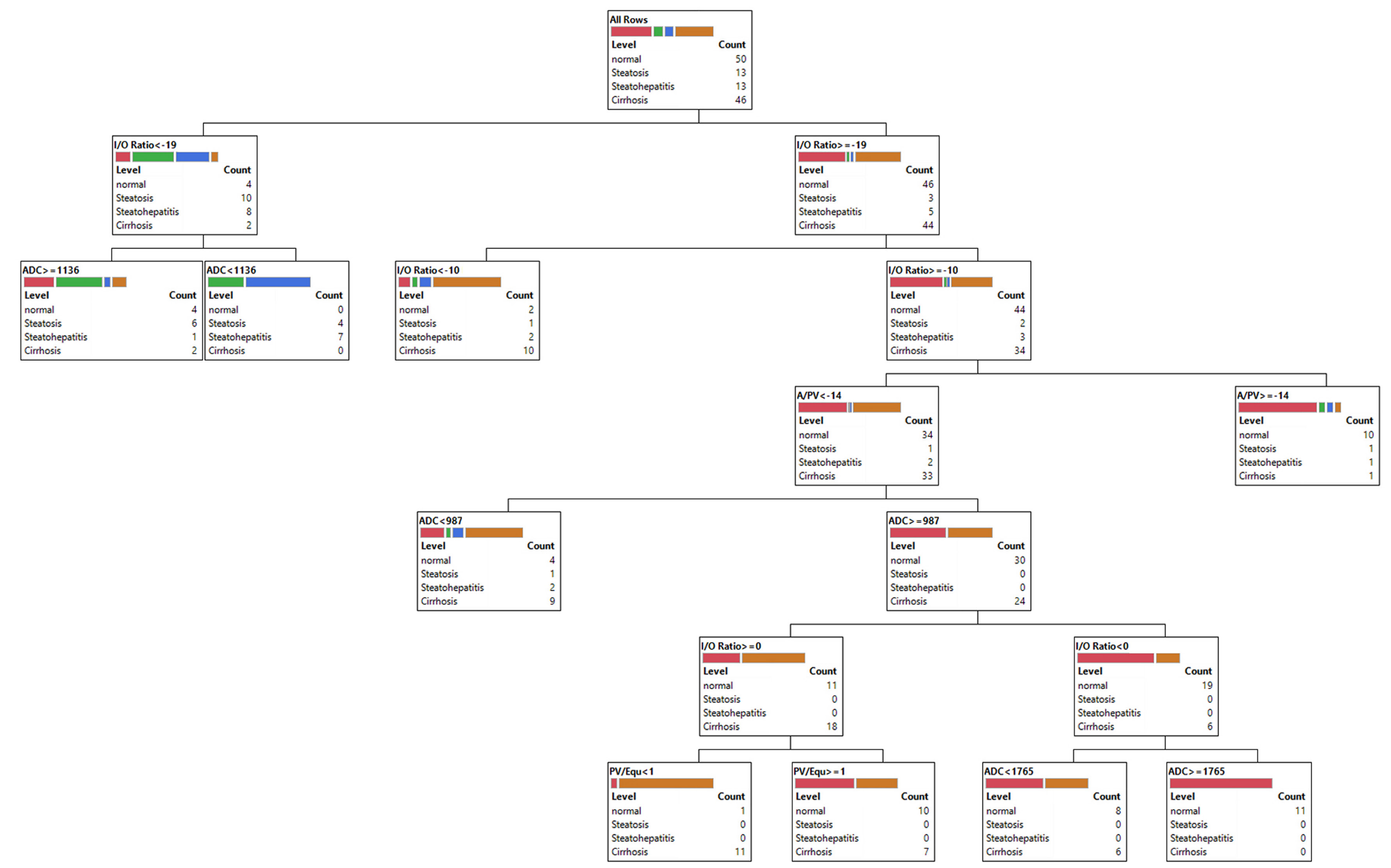

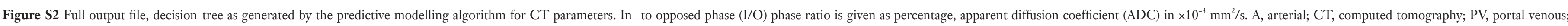
Equ, equilibrium contrast enhancement phase. 